\title{
Disordered Kondo nanoclusters: Effect of energy spacing
}

\author{
C. Verdozzi,* Y. Luo, and Nicholas Kioussis \\ Department of Physics, California State University Northridge, Northridge, California 91330-8268, USA
}

(Received 4 May 2004; published 14 October 2004)

\begin{abstract}
Exact-diagonalization results for Kondo nanoclusters alloyed with mixed-valence impurities show that tuning the energy spacing $\Delta$ drives the system from the Kondo to the Ruderman-Kittel-Kasuya-Yosida (RKKY) regime. The interplay of $\Delta$ and disorder gives rise to a $\Delta$ versus concentration $T=0$ phase diagram very rich in structure. The parity of the total number of electrons alters the competition between the Kondo and RKKY correlations. The local Kondo temperatures $T_{K}$ and RKKY interactions depend strongly on the local environment and overall are enhanced by disorder, in contrast to the hypothesis of "Kondo disorder" single-impurity models.
\end{abstract}

DOI: 10.1103/PhysRevB.70.132404

PACS number(s): 75.20.Hr, 75.40.Cx, 75.40.Mg, 75.75.+a

Magnetic impurities in nonmagnetic hosts have been one of the central subjects in the physics of strongly correlated systems for the past four decades. ${ }^{1}$ Such enduring, ongoing research effort is motivated by a constant shift and increase of scientific interest over the years, from dilute ${ }^{2}$ to concentrated impurities, ${ }^{3}$ from periodic ${ }^{4}$ to disordered samples, ${ }^{5,6}$ and from macroscopic ${ }^{7}$ to nanoscale phenomena. ${ }^{8}$

Various disorder-driven models have been proposed to explain the experimentally observed ${ }^{7}$ non-Fermi-liquid (NFL) behavior at low temperatures. ${ }^{5-7,9}$ The phenomenological "Kondo disorder" approaches, ${ }^{5,10}$ based on single-impurity models, assume a distribution of Kondo temperatures due to a distribution of either $f-c$ orbital hybridization or of impurity energy levels. In such models, the presence of certain sites with very low $T_{K}$ leads to a NFL behavior at low $T$. An open issue in such methods is whether including RudermanKittel-Kasuya-Yosida (RKKY) interactions would renormalize and eliminate the low- $T_{K}$ spins. An alternative view is the formation of large but finite magnetic clusters (Griffith phases) within the disordered phase through the competition between the RKKY and Kondo interactions., 6,11

The relevance of small strongly correlated systems to quantum computation requires understanding how the infinite-size properties become modified at the nanoscale, due to the finite energy spacing $\Delta$ in the conduction band..$^{8,12-15}$ Recent scanning tunneling microscope (STM) experiments ${ }^{16}$ studied the interaction of magnetic impurities with the electrons of a single-walled nanotube confined in one dimension: In addition to the bulk Kondo resonance new subpeaks were found in shortened carbon nanotubes, separated by about the average energy spacing in the nanotube. For such small systems, controlling $T_{K}$ upon varying $\Delta$ is acquiring increasing importance since it allows us to tune the cluster magnetic behavior and to encode quantum information. While the effect of $\Delta$ on the single-impurity Anderson or Kondo model has received considerable theoretical ${ }^{8,12-15}$ and experimental ${ }^{16}$ attention recently, their role on dense impurity clusters with or without disorder remains an unexplored area thus far.

In this work we present exact diagonalization calculations for $d$ - or $f$-electron nanoclusters to study the effect of disorder and $\Delta$ on the interplay between the Kondo and RKKY interactions. While the cluster properties depend on cluster geometry and size,${ }^{17}$ the present calculations treat exactly the Kondo and RKKY interactions, the disorder averages, and provide a distribution of local $T_{K}$ 's renormalized by the intersite $f$ - $f$ interactions. Our results show that (i) tuning $\Delta$ and/or the parity of the total number of electrons $N_{e l}$ can drive the nanocluster from the Kondo to the RKKY regime, i.e., a Doniach phase diagram ${ }^{18}$ in small clusters; (ii) the $\Delta$ versus alloy concentration $T=0$ phase diagram exhibits regions with prevailing Kondo or RKKY correlations alternating with domains of ferromagnetic (FM) order; and (iii) the local $T_{K}$ 's and the nearest-neighbor (NN) RKKY interactions depend strongly on the local environment and are overall enhanced by disorder. This enhancement of $T_{K}$ in the disordered clusters is in contrast to the hypothesis of "Kondo disorder" models for extended systems.

We consider a random binary alloy cluster, $A_{N-x} B_{x}$, of $N$ $=6$ sites and a different number of $B$ atoms, $x=0-N$, arranged in a ring described by the half-filled $\left(N_{e l}=2 N=12\right)$ two-band lattice Anderson Hamiltonian,

$$
\begin{aligned}
H= & t \sum_{\langle i j\rangle \sigma} c_{i \sigma}^{\dagger} c_{j \sigma}+\sum_{i \sigma} \epsilon_{f}^{i} f_{i \sigma}^{\dagger} f_{i \sigma}+\sum_{i} U_{i} f_{i+}^{\dagger} f_{i+} f_{i-}^{\dagger} f_{i-}+\sum_{i \sigma} V\left(f_{i \sigma}^{\dagger} c_{i \sigma}\right. \\
& \left.+c_{i \sigma}^{\dagger} f_{i \sigma}\right) .
\end{aligned}
$$

Effect of disorder. We introduce binary disorder in the $f$-orbital energy $\epsilon_{f}^{i}\left(=\epsilon_{f}^{A}\right.$ or $\left.\epsilon_{f}^{B}\right)$ and in the intra-atomic Coulomb energy $U_{i}\left(=U^{A}\right.$ or $\left.U^{B}\right)$ to model a Kondo-type $A$ atom with $\epsilon_{f}^{A}=-U^{A} / 2=-3$ (symmetric case) and a mixed-valent (MV) type $B$ atom with $\epsilon_{f}^{B}=-2$ and $U^{B}=1$. For both types of atoms $V^{A}=V^{B}=V=0.25$. For $t=1$, this choice of parameters leads to a degeneracy between the doubly degenerate $c$-energy levels, $\epsilon_{k}=-t$, and the energy level $\epsilon_{f}^{B}+U^{B}$. Upon filling the single particle energy levels for any $x, N-x(x)$ electrons fill the $\epsilon_{f}^{A}\left(\epsilon_{f}^{B}\right)$ levels, and two electrons fill the $-2 t$ conduction energy level, with the remaining $N-2$ electrons accommodated in the $x+4$ degenerate states at $-t$. This in turn results in strong charge fluctuations.

The nonequivalent configurations for $x \leqslant 3$ are shown in Fig. 1, left panel, along with the value of the spin, $S_{g}$, of the ground state for $t=1$. Except for the cases $x=0,5,6$, with a $S_{g}=0$ ground state, for all other $x$ there are configurations with $S_{g} \neq 0$. The average occupation and average local mo- 

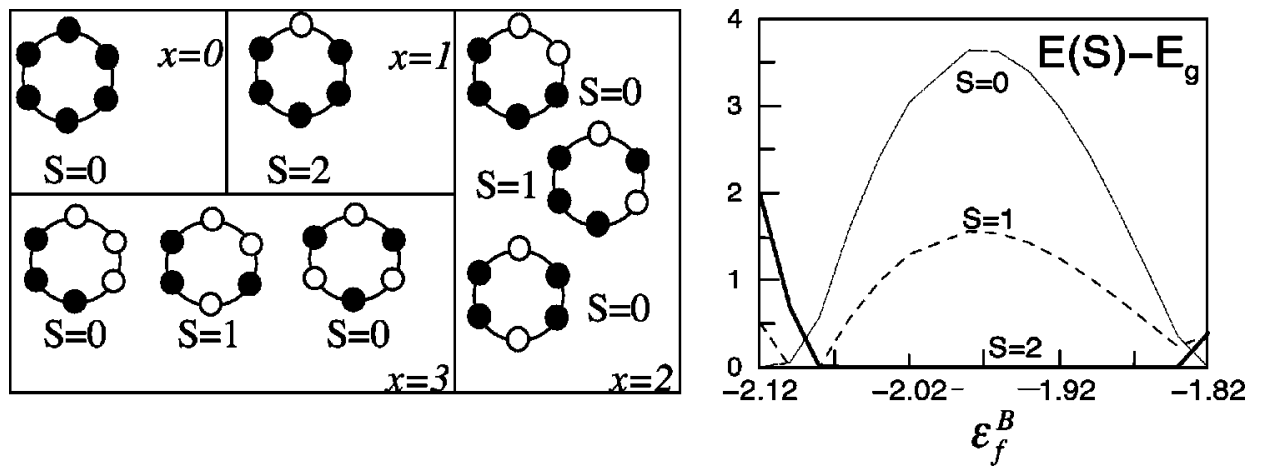

FIG. 1. Left panel: Alloy configurations for various concentrations $x \leqslant 3$ along with the value of the spin $S_{g}$ in the ground state for $t=1$. Right panel: Energy difference (in units of $10^{-4} t$ ) between the lowest eigenstates and the ground state as a function of $\epsilon_{f}^{B}$. ment (LM) for the periodic Kondo and MV lattices are $\left\langle n_{f}^{A}\right\rangle=1,\left\langle\left(\mu_{f}^{A}\right)^{2}\right\rangle=0.99$, and $\left\langle n_{f}^{B}\right\rangle=1.6,\left\langle\left(\mu_{f}^{B}\right)^{2}\right\rangle=0.43$, respectively. We carry out a detailed analysis for $x=1\left(S_{g}=2\right)$ to demonstrate the FM transition induced by a single MV atom in an otherwise Kondo cluster. Studies of extended systems reported a similar occurrence of ferromagnetism in the MV phase. ${ }^{19}$ As expected, the singlet ground state of the $x=0$ Kondo cluster is characterized by $\mathrm{NN}$ antiferromagnetic (AF) $f-f$ spin correlations at $T=0$, i.e., $\left\langle S_{f}^{A}(i) S_{f}^{A}(i+1)\right\rangle$ $\equiv\left\langle g\left|S_{z, f}^{A}(i) S_{z, f}^{A}(i+1)\right| g\right\rangle=-0.58$, where $|g\rangle$ is the many-body ground state and $S_{z, f}^{A}$ is the $z$ component of the $f$ spin of the $\mathrm{A}$ atom. Introducing a MV atom renders them FM. Since $U^{B}=1$, the $B$ impurity tends to remove charge from the $k$ state with $\epsilon_{k}=-t$, which has large amplitude at the $B$ site and at the opposite $A$ site across the ring. Such a depletion is different for the two spin states, yielding a maximum value for the $f$ moment of the MV atom. The $f-f$ spin correlation function between the Kondo and $\mathrm{MV}$ atoms are $\mathrm{AF}$ $\left[\left\langle S_{f}^{A}(i) S_{f}^{B}(i+1)\right\rangle=-0.23\right]$, while they are FM among the Kondo atoms $\left[\left\langle S_{f}^{A}(i) S_{f}^{A}(i+1)\right\rangle=+0.94\right]$. A similar result was recently found in $a b$ initio calculations, ${ }^{20}$ where a nitrogen impurity in small Mn clusters induces ferromagnetism via AF coupling between the $\mathrm{N}$ to the $\mathrm{Mn}$ atoms, while $\mathrm{Mn}$ -Mn couple ferromagnetically. However, the physical system, methodology, and mechanism in this work are vastly different from those of Ref. 20. Namely, the densityfunctional theory approach would fail to reproduce the Kondo effect in strongly correlated nanoclusters. We find that there is a crossover in $S_{g}$ from $0 \rightarrow 1 \rightarrow 2 \rightarrow 0$ (Fig. 1, right panel), indicating a reentrant nonmagnetic transition around $\epsilon_{f}^{B}=2$. This almost saturated FM domain is robust against small changes in $U^{B}, V, \epsilon_{f}^{A}, U^{A}$, cluster size $(N=7)$, and band filling $\left(N_{e l}=10\right)$ provided that the Kondo atom has a large LM.

We have also calculated the temperature-dependent average susceptibility, $\chi_{x}^{f}(T)$, at concentration $x$,

$$
\frac{k_{B} T \chi_{x}^{f}(T)}{\left(g \mu_{B}\right)^{2}}=\frac{1}{Q} \sum_{C_{x}, \alpha_{C_{x}}} e^{-E_{\alpha_{C_{x}}}{ }^{\prime} k_{B} T}\left\langle\alpha_{C_{x}}\left|S_{f}(i) S^{T o t}\right| \alpha_{C_{x}}\right\rangle .
$$

Here, $S^{T o t}$ is the $z$ projection of the total spin (both the $f$ and $c$-contributions), $C_{x}$ denote the configurations at concentration $x,\left|\alpha_{C_{x}}\right\rangle$ and $E_{C_{x}}$ are the configuration-dependent exact many-body eigenstates and eigenvalues, respectively, and $Q$ denotes the partition function. In Fig. 2 we present $T \chi_{x}^{f}(T)$ as a function of temperature for different $x$. As $T \rightarrow 0$ (inset Fig. 2) $T \chi_{x}^{f}(T)$ approaches a finite value for $x=1-4$ while it vanishes exponentially for $x=0,5$, and 6 . This is because the former concentrations involve some configurations which are magnetic, while the latter have singlet ground states (Fig. 1). The stronger (weaker) low-temperature dependence for $x$ $=1(x=2-4)$ is due to the smaller (larger) spin gap between the ground state and the lowest excited states. The magnetic susceptibility displays also a magnetic crossover upon varying $x$, and reveals a Curie-like divergence at low $T$ for $x=1$ -4 . The temperature-dependent specific heat, not reported here, shows corroborative evidence of such crossover.

$R K K Y$ versus Kondo interactions: Effect of $\Delta$ and parity. Next we examine (1) the effect of $\Delta$ and of the parity of $N_{e l}$ on the interplay between RKKY and Kondo interactions, (2) the characterization of the single-impurity "Kondo correlation energy" $T_{K}$ in a dense-impurity cluster, and (3) the effect of disorder and $\Delta$ on the distribution of the local $T_{K}$ 's. In the following, $\epsilon_{f}^{B}=-2$.

In contrast with previous studies, ${ }^{5}$ which introduced a phenomenological distribution of local single-impurity $T_{K}(i)$ 's, we compute exactly the Kondo correlation energy using the "hybridization" approach: ${ }^{21}$

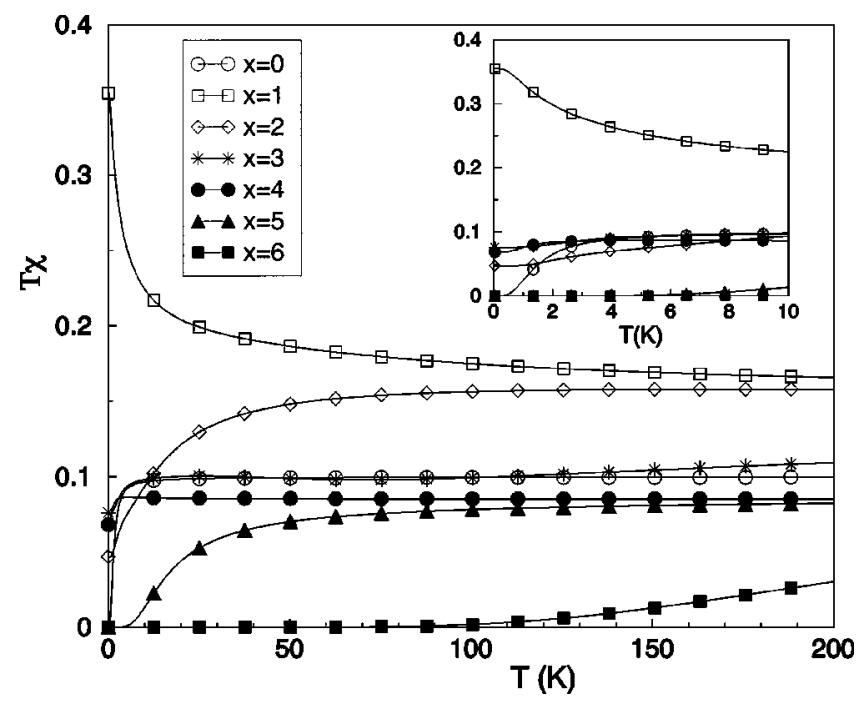

FIG. 2. Temperature dependence of the average $f$ susceptibility for different alloy concentrations. The inset shows the lowtemperature behavior. 
TABLE I. Local $f$ - $c$ and NN $f$ - $f$ SCF's and local Kondo temperature (in $\mathrm{K}$ ) for two values of $t$.

\begin{tabular}{cccc}
\hline \hline $\mathrm{t}(\mathrm{eV})$ & $\left\langle S_{f}^{A}(i) S_{c}^{A}(i)\right\rangle$ & $\left\langle S_{f}^{A}(i) S_{f}^{A}(i+1)\right\rangle$ & $T_{K}(i)$ \\
\hline 0.05 & -0.626 & -0.322 & 551.8 \\
1.00 & -0.011 & -0.584 & 173.4 \\
\hline \hline
\end{tabular}

$$
k_{B} T_{K}(i)=E_{g}\left(V_{i}=0\right)-E_{g} .
$$

Here, $E_{g}\left(V_{i}=0\right)$ is the ground-state energy of the denseimpurity cluster when $V$ is set to zero at the $i$ th site. Equation (3) reduces to $k_{B} T_{K}=E_{\text {band }}-E_{F}+\epsilon_{f}-E_{g}$ (Refs. 13 and 22) in the single-impurity case, where $E_{F}$ is the highest occupied energy level in the conduction band and $E_{\text {band }}$ is the conduction band energy. This definition of $T_{K}(i)$ takes into account the intersite $f$ - $f$ interactions.

In Table I we list for the $x=0$ case the local Kondo $f$ - $c$ spin correlation function $\left\langle S_{f}^{A}(i) S_{c}^{A}(i)\right\rangle$, the NN $f$ - $f$ spin correlation function $\left\langle S_{f}^{A}(i) S_{f}^{A}(i+1)\right\rangle$, and $T_{K}(i)$ for two different values of $t$ [the energy spacing is $\Delta=4 t /(N-1) \equiv 4 t / 5$ ]. As $t$ or $\Delta$ decreases the $f-c$ spin correlation function (SCF) is dramatically enhanced while the $f$ - $f$ correlation function becomes weaker, indicating a transition from the RKKY to the Kondo regime. This is also corroborated by the increase in $T_{K}(i)$. The energy spacing affects not only the magnetic $(A)$ atoms but the MV atoms as well. Thus, increasing $t$ drives the $B$ atoms from the nonmagnetic (NM) $\left(n_{f} \approx 2\right)$ to the MV and finally to the Kondo regime.

We next examine the effect of changing $N_{e l}$ from $N_{e l}$ $=12\left(S_{g}=0\right)$ to $N_{e l}=11\left(S_{g}=\frac{1}{2}\right)$ for $t=1$ and $x=0$. We find (a) an enhancement of the local Kondo $f$-c SCF $\left\langle S_{f}^{A}(i) S_{c}^{A}(i)\right\rangle$ from -0.01 to -0.12 , and (b) a suppression of the NN $f$ - $f$ $\operatorname{SCF}\left\langle S_{f}^{A}(i) S_{f}^{A}(i+1)\right\rangle$ from -0.58 to -0.20 (due to the broken symmetry for $N_{e l}=11$, the $f-f$ SCF's range from -0.5 to +0.02 ). This interesting novel tuning of the magnetic behavior can be understood in terms of the (single versus double) topmost occupied conduction level: For $N_{e l}$ even, double occupancy prevents spin-flip transitions, thus weakening the the Kondo correlations. ${ }^{12}$ We found similar results for smaller $t$ and for $x>0$.

In Fig. 3 we present the local $T_{K}(i)$ versus the $T=0$ configuration-dependent local $f$-c $\quad \mathrm{SCF}, \quad\left\langle S_{f}^{A}(i) S_{c}^{A}(i)\right\rangle$ $\equiv\left\langle g_{C_{x}}\left|S_{z, f}^{A}(i) S_{z, c}^{A}(i)\right| g_{C_{x}}\right\rangle$, for all concentrations $x$ and for $t$ $=0.05$ and 1.0 (we considered only $S_{g}=0$ configurations). Note the different scales both on the horizontal and vertical axis in the panels. In both panels, the closed circles correspond to the $x=0$ case. The results indicate a correlation between $T_{K}$ and the $f$ - $c$ SCF (the larger $T_{K}$ 's correspond to the more negative $f$-c values) as one would expect, since both provide a measure of the Kondo effect. For $t=0.05$, most of the disordered cluster configurations are in the Kondo regime $\left(S_{g}=0\right)$ with larger $T_{K}$ values; consequently, panel (a) has a larger number of singlet configurations. The introduction of MV impurities induces a distribution of $T_{K}(i)$ 's, whose values are overall enhanced compared to those for the $x=0$ case, in contrast with single-site theories for extended systems. ${ }^{5}$ This enhancement may be due to the more localized character of the conduction electron states in the presence of disorder. It is interesting that the distribution of $T_{K}$ 's for $t=0.05$ exhibits a bimodal behavior centered about 710 and $570 \mathrm{~K}$, respectively: The higher $T_{K}$ 's originate from isolated Kondo atoms which have MV atoms as NN's so that the local screening of the magnetic moment of the $A$ atom is enhanced.

The effect of alloying and $\Delta$ on the RKKY versus Kondo competition for a given $x$ is seen in Fig. 4 (left panel), where the $T=0$ configuration averaged SCF's, $\left\langle S_{f}^{A}(i) S_{c}^{A}(i)\right\rangle_{x}$ $\equiv N_{C_{x}}^{-1} \Sigma_{C_{x}}\left\langle g_{C_{x}}\left|S_{z, f}^{A}(i) S_{z, c}^{A}(i)\right| g_{C_{x}}\right\rangle$ and $\left\langle S_{f}^{A}(i) S_{f}^{A}(i+1)\right\rangle_{x}$, are plotted versus $t$. The solid curves denote the $x=0$ case, where we drive the cluster from the RKKY to the Kondo regime as we decrease $t$. We find that the stronger the average Kondo correlations are, the weaker the average RKKY interactions and vice versa. In the RRKY regime $(t \gg 1)$, the configurationaveraged RKKY SCF's for different concentrations are widely distributed (in the range between -0.6 and -1 ) about the $x=0$ curve, indicating that they are sensitive to the local environment. In contrast, in the Kondo regime $(t \ll 1)$, the Kondo atoms become locked into singlets and the RKKY correlations are clustered around the $x=0$ curve, i.e., they are insensitive to the local environment. Interestingly, both $\Delta$ and disorder lead to an enhancement of the RKKY interactions compared to the uniform state.

In the right panel of Fig. 4 we present the $t$ versus $x$ phase diagram for the nanocluster at $T=0$, which illustrates the evolution of magnetic order and competition between Kondo and RKKY interactions. We compare the configuration-

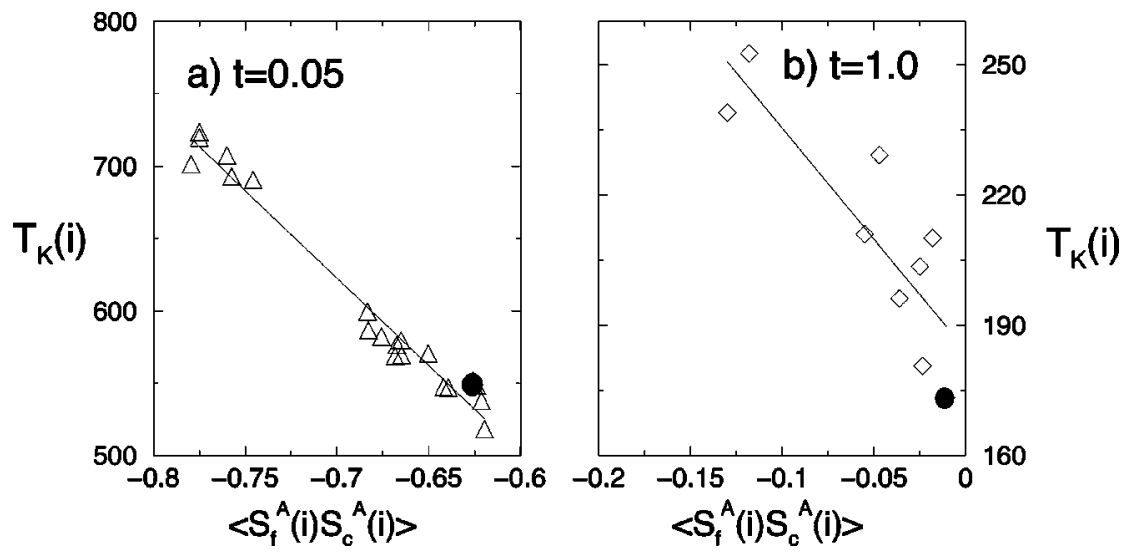

FIG. 3. $A$ atoms: Local Kondo temperatures (in $\mathrm{K}$ ) vs the local $f$ $-c$ SCF for different configurations and two values of $t$. The closed circles refer to the $x=0$ case and the lines are a guide to the eye. 


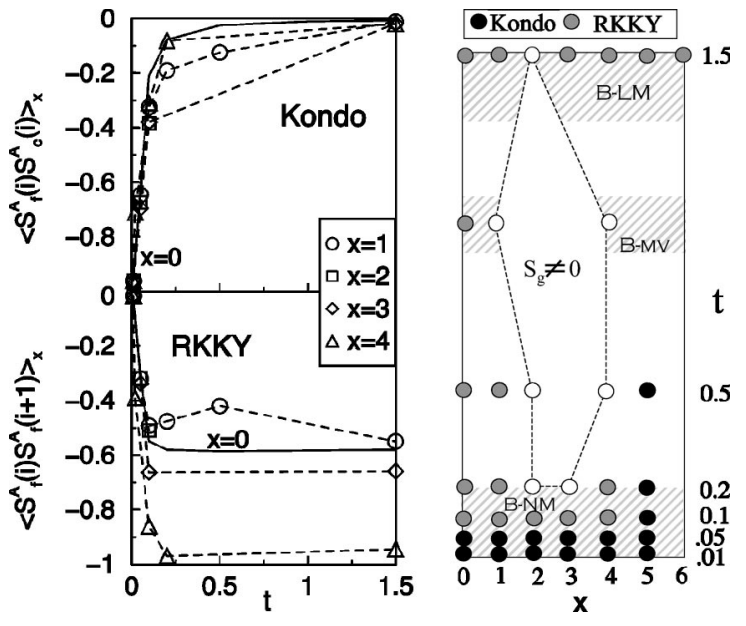

FIG. 4. Left panel: Configuration-averaged local $f$-c (top) and NN $f$ - $f$ SCF's (bottom) for the A atoms as a function of $t$. The solid line denotes the $x=0$ case. Right panel: Zero-temperature $t$ vs $x$ phase diagram for the nanocluster. Black (gray) circles denote the Kondo (RKKY) regime. The white circles and the dashed contour delimit the FM region. The horizontal gray stripes denote qualitatively regions of NM, MV, and LM behavior of the $B$ atoms.

averaged $\left\langle S_{f}^{A}(i) S_{c}^{A}(i)\right\rangle_{x}$ and $\left\langle S_{f}^{A}(i) S_{f}^{A}(i+1)\right\rangle_{x}$ to assign a state of specific concentration to the Kondo or RKKY regimes (black and gray circles, respectively), in analogy with the $x$ $=0$ case and with mean field treatments. ${ }^{23}$ An interesting feature of the phase diagram is the appearance of a large FM region $\left(S_{g} \neq 0\right)$ enclosed by the dashed line. The RKKY region at large $t$ and large $x$ originates from the $\mathrm{B}$ atoms which become magnetic. For the non-FM configurations and for $x<5$ the Kondo (RKKY) correlations of the $A$ atoms dominate at small (large) $t$, in analogy with the $x=0$ case. On the other hand, for $x=5$ the local Kondo correlations of the single $A$ atom at low $t$ dominate over the $f$ - $f$ correlations between the $A-B$ and $B-B$ pairs. For the uniform $(x=6) \mathrm{MV}$ case we include only results in the large $t$ regime, where the MV atoms acquire LM's which couple antiferromagnetically. Overall, the RKKY interactions prevail for any $x$ when $t$ $\geqslant V$.

In conclusion, exact diagonalization results reveal that the energy spacing, disorder, and parity of $N_{e l}$ give rise to an interesting tuning of the magnetic behavior of a dense Kondo nanocluster. This interesting and important tuning can drive the nanocluster from the Kondo to the RKKY regime, i.e., a tunable Doniach phase diagram at the nanoscale. The interplay of $\Delta$ and disorder produces a zero-temperature alloy phase diagram very rich in structure. The distribution of local $T_{K}$ 's and RKKY interactions depends strongly on the local environment and are overall enhanced by disorder, in contrast to the hypothesis of single-impurity based "Kondo disorder" models for extended systems. Our conclusions should be relevant to experimental realizations ${ }^{16}$ of small clusters and quantum dots, with appropriate tuning of the energy spacing.

We acknowledge useful discussions with P. Fulde, P. Schlottmann, P. Riseborough, A.H. Castro Neto, P. Cornaglia, and C. Balseiro. The research was supported through the NSF under Grant Nos. DMR-0097187 and DMR0011656, NASA under grant No. NCC5-513, and the Keck and Parsons Foundations grants.
*Electronic address: claudio.verdozzi@ csun.edu

${ }^{1}$ A. C. Hewson, The Kondo Problem to Heavy Fermions, Cambridge Press, New York, 1993.

${ }^{2}$ P. W. Anderson, Phys. Rev. 124, 41 (1961); J. Kondo, Prog. Theor. Phys. 32, 37 (1964).

${ }^{3}$ C. D. Bredl, S. Horn, F. Steglich, B. Luthi, and R. M. Martin, Phys. Rev. Lett. 52, 1982 (1984).

${ }^{4}$ H. Tsunetsugu, M. Sigrist, and K. Ueda, Rev. Mod. Phys. 69, 809 (1997).

${ }^{5}$ E. Miranda, V. Dobrosavljevic, and G. Kotliar, Phys. Rev. Lett. 78, 290 (1997).

${ }^{6}$ A. H. Castro Neto and B. A. Jones, Phys. Rev. B 62, 14975 (2000).

${ }^{7}$ G. R. Stewart, Rev. Mod. Phys. 73, 797 (2001).

${ }^{8}$ P. Schlottmann, Phys. Rev. B 65, 174407 (2002).

${ }^{9}$ P. S. Riseborough, Phys. Rev. B 45, 13984 (1992).

${ }^{10}$ O. O. Bernal, D. E. MacLaughlin, H. G. Lukefahr, and B. Andraka, Phys. Rev. Lett. 75, 2023 (1995).

${ }^{11}$ E. Miranda and V. Dobrosavljevic, Phys. Rev. Lett. 86, 264
(2001).

${ }^{12}$ W. B. Thimm, J. Kroha, and J. von Delft, Phys. Rev. Lett. 82, 2143 (1999).

${ }^{13}$ H. Hu, G. M. Zhang, and L. Yu, Phys. Rev. Lett. 86, 5558 (2001).

${ }^{14}$ P. S. Cornaglia and C. A. Balseiro, Phys. Rev. B 66, 115303 (2002).

${ }^{15}$ P. Simon and I. Affleck, Phys. Rev. Lett. 89, 206602 (2002).

${ }^{16}$ T. Odom, J. L. Huang, C. Li Cheung, and C. M. Lieber, Science 290, 1549 (2000) and references therein.

${ }^{17}$ G. M. Pastor, R. Hirsch, and B. Mühlschlegel, Phys. Rev. Lett. 72, 3879 (1994).

${ }^{18}$ S. Doniach, Physica B \& C 91, 231 (1977).

${ }^{19}$ D. Meyer and W. Nolting, Phys. Rev. B 62, 5657 (2000).

${ }^{20}$ B. K. Rao and P. Jena, Phys. Rev. Lett. 89, 185504 (2002).

${ }^{21} \mathrm{P}$. Fulde (private communication); P. Fulde, Electron Correlations in Molecules and Solids, 3rd ed. (Springer, Berlin, 1995).

${ }^{22}$ K. Yosida, Phys. Rev. 147, 223 (1966).

${ }^{23}$ B. Coqblin, C. Lacroix, M. S. Gusmao, and J. R. Iglesias, Phys. Rev. B 67, 064417 (2003). 